\title{
Development of algorithms for hand movements classification based on optical fiber force myography signals
}

\section{Willian H. A. da Silva*, Matheus K. Gomes, Eric Fujiwara}

\begin{abstract}
Force myography (FMG) is the mechanical counterpart of the surface electromyography, providing a low-cost and straightforward alternative for tracking the hand movements in applications related to rehabilitation and robotics. This project proposes the development of an algorithm for classification of hand postures based on the FMG signals measured by an optical fiber sensor, with artificial neural networks design and implementation in embeeded system.
\end{abstract}

\section{Key words:}

Force myography, optical fiber sensor, artificial neural networks

\section{Introduction}

The assessment of the hand movements is important for several fields, such as robotics, medicine, entertainment and assistive technologies. The classification of postures by myographic signals analysis can be carried out by machine learning and auto-regressive moving average algorithms $^{1}$, but the lack of modularization and the limitation to specific operational systems limit their use in practical applications.

In this work, an optical fiber FMG sensor ${ }^{2}$ was tested for identifying up to 9 hand postures. The system is comprised of multimode fiber, LED source, 3D-printed force transducer, photodetector, and a Raspberry $\mathrm{Pi}$ microcomputer.

\section{Results and Discussion}

The classification algorithm was implemented using open source technologies and libraries. The intensity signals from the fiber are subjected to preprocessing tasks, such as normalizing, windowing, and filtering, using a Python script and a low pass Butterworth filter. Three of the preprocessed output signals are shown in Fig. 1.

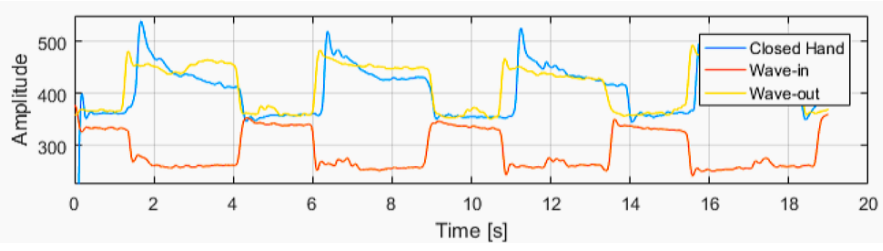

Fig. 1. Characteristic signals for three different hand postures used for the training process.

For the classification pipeline, several ANN architectures were implemented, designing one of them for optimized performance in steady-state signal classification, which is related to multiple FMG channels, and the other optimized for time-domain signals, which is suitable to single-channel classification, as shown in Fig. 2.

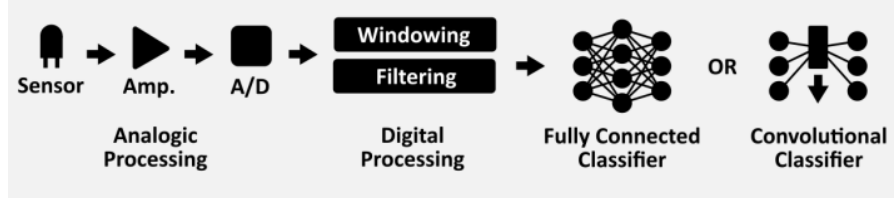

Fig. 2. Processing diagram for hardware and software implementation of the classifier.
As a result of the process, the confusion matrices related to each classification process are shown in Fig. 3 along with the transducer used with the FMG sensor in Fig. 4.

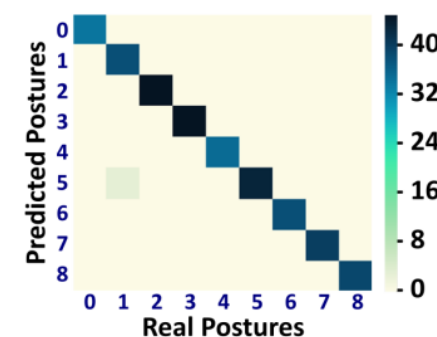

(a)

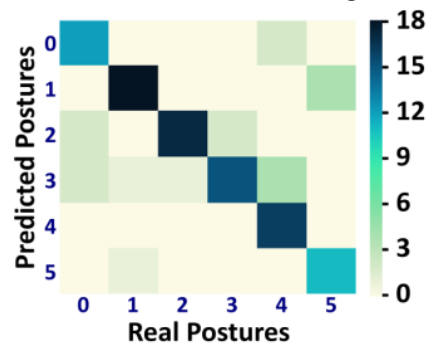

(b)
Fig. 3. Confusion matrix generated for (a) the fully connected ANN model and (b) the convolutional model.

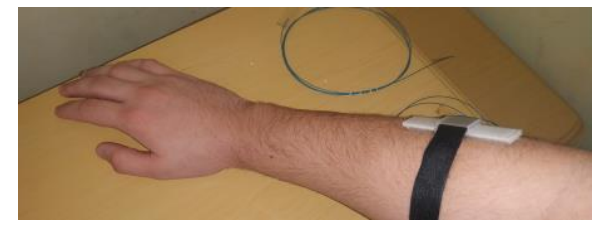

Fig. 4. Force transducer used in the user's forearm.

The first architecture provided $99.5 \%$ accuracy and average error of $0.53 \pm 0.09 \%$ verified with the $\mathrm{k}$-fold cross validation method $^{3}$. Moreover, the second architecture was capable to identify 6 hand postures with a single FMG channel, yielding 82.4\% accuracy evaluated directly by the Tensorflow library module.

\section{Conclusions}

Algorithms for hand posture classification based on ANN were successfully designed for processing optical fiber FMG signals. The algorithm can be embedded in portable hardware for implementing costumer-oriented FMG sensors, with possible applications in rehabilitation and human-computer interaction.

\section{Acknowledgement}

This work was supported in parts by CNPq/PIBIC and FAPESP under grant 2017/25666-2.

\footnotetext{
1 Taşar, B. et. al.; EMG-Controlled Prosthetic Hand with Fuzzy Logic Classification Algorithm, Modern Fuzzy Control Systems and Its Applications, IntechOpen, 2017.

${ }^{2}$ Fujiwara, E.; Suzuki, C. K.; Journal of Sensors, 2018.

${ }^{3}$ Rodriguez, J. D.; Perez, A.; Lozano, J. A.; IEEE Trans. Pattern Anal. Machine Intell., vol. 32, no. 3, pp. 569-575, 2010.
} 